\title{
PREDICTION OF FORMING LIMIT DIAGRAMS OF AL-MG ROLLED SHEETS TAKIIVG TEXTURE INTO ACCOUNT
}

\author{
P. RATCHEV ${ }^{1}$, P. VAN HOUTTE ${ }^{1}$, B. VERLINDEN ${ }^{1}$, P. DE SMET ${ }^{2}$, \\ P. NEUTJENS ${ }^{2}$, R. BAARTMAN ${ }^{3}$ AND P. DRENT ${ }^{3}$ \\ ${ }^{1}$ Katholieke Universiteit Leuven, Department MTM, de Croylaan 2, \\ 3001 Leuven, Belgium \\ ${ }^{2}$ Hoogovens Aluminium N. V., Quality Department, A Stocletlaan 87, \\ 2570 Duffel, Belgium \\ ${ }^{3}$ Hoogovens Corporate Research, P.O. Box 10000, 1970-CA IJmuiden,
} The Netherlands

(Received 24 February 1994)

\begin{abstract}
Forming limit diagrams of an AA5754 Al-Mg alloy have been calculated, taking the sheet texture into account. An approach based on the Marciniak-Kuszynski defect model for calculating forming limit diagrams (FLD's) has been used. The influence of different initial parameters on the formability curve has been studied. The influence of different ideal texture components on simulated curves has been investigated as well. A comparison between the simulated and the measured FLD is given and critically discussed.
\end{abstract}

KEY WORDS: Texture, formability, Al-Mg alloy, rolling.

\section{INTRODUCTION}

The pressure on the automotive industry to produce lighter cars with reduced fuel consumption causes a demand for new materials able to replace steel for car body panels. Some of the materials considered for this are the aluminium alloys, especially $\mathrm{Al}-\mathrm{Mg}$ alloys of the 5000 series. Although they combine a reasonable strength with fair corrosion resistance and formability, their mechanical properties are still inferior to those of steel and further improvement is required. Especially important is an improvement of the formability because on average, the limit strain of aluminium alloys in the plane strain region is only half that of steel.

Forming limit diagrams (FLD's) are an important tool for sheet formability investigations. In a FLD, the limit strains of a sheet are plotted for a wide range of strain paths. Since the experimental measurement of a FLD is rather time consuming, a substantial effort has recently been made to develop reliable numerical codes predicting FLD's for metal sheets, see for example Ferron and Molinari (1989) or Graf and Hosford (1989). An other driving force for the development of computer models is the possibility to study the influence of different initial parameters on the FLD, which is important regarding material properties improvement. 
The aim of the present paper is to predict the FLD of a commercial Al-3 wt.\% $\mathrm{Mg}$ alloy, taking the sheet texture into account, and to compare it to the measured FLD. A recently developed method based on the Marciniak-Kuszynski defect model for calculating forming limit diagrams has been used (Van Houtte and Toth, 1993). The influence of different initial parameters on the formability curve has been studied, with special emphasis on texture. The influence of some ideal texture components on simulated curves has been studied for this purpose.

\section{EXPERIMENTS AND CALCULATIONS}

The FLD simulations have been done by means of a recently developed numerical procedure based on the Marciniak-Kuszynski defect model (Van Houtte and Toth, 1993) which takes the specimen anistropy into account. The textures of the samples have been measured by means of X-ray diffraction. Four pole figures, namely $\{111\}$, $\{200\},\{220\}$ and $\{311\}$, have been measured in the middle layer of the samples $(50 \%$ of the samples thickness having been removed) by the Schulz back-reflection method. These pole figures were converted into an orientation distribution function (ODF) using the harmonic method of Bunge (1982) (analysis of incomplete pole figures leading to even C-coefficients and a ghost correction procedure). The volume fractions of the texture components have been generated by a deconvolution method. The yield surface, necessary for the prediction of FLD's, was constructed from the generated ODF using crystallographic slip and the Taylor polycrystal model (Van Houtte, Mols, Van Bael and Aernoudt, 1989).

The important initial parameters used in the simulations, according the MarciniakKuszynski (1967) defect model are:

- the strain rate sensitivity exponent $m$,

- the strain hardening coefficient $n$,

- the input textures, by means of C-coefficients, describing the ODF,

- the initial groove thickness.

The influence of every one of them is considered separately. The Marciniak theory assumes that initially, there are grooves with various orientations present in the material. The model then calculated how the depth of such grooves evolves during ongoing deformation. In principle, the limit strain is the strain of the material outside the groove at the time that the depth of groove becomes equal to half of the thickness of the sheet outside the groove becomes equal to half of the thickness of the sheet outside the groove. Hence the Marciniak model essentially calculates the evolution of ductile fracture. This theory needs the depth of the initial groove (of the thickness of the sheet at the groove) as an initial parameter.

The experimentally measured FLD's have produced by an optimised Nakasima method (Taylor, 1985). Halter-shaper blanks were stretch formed over a $75 \mathrm{~mm}$ spherical punch on an Erichsen laboratory press. Minor strains were controlled by changing the width of the halter-shaped section. A $2 \mathrm{~mm}$ circular grid, applied by means of fotochemical etching, was used to measure the strains after deformation. A curve was calculated from the strain data using non-linear regression techniques.

The test were done on cold rolled sheets of a commercially available $\mathrm{Al}-\mathrm{Mg}$ alloy of type AA 5754 with $3 \mathrm{wt} \% \mathrm{Mg}, 0.25 \mathrm{wt} \% \mathrm{Mn}, 0.2 \mathrm{wt} \% \mathrm{Si}$ and $0.35 \mathrm{wt} \% \mathrm{Fe}$. The material was stretcher strain free due to the controlled coarse grain size received by 
Table 1 Texture components and volume fractions (of the respective ideal texture component) of the measured aluminium alloy.

\begin{tabular}{|c|c|c|}
\hline Texture component & l & Volume fraction (\%) \\
\hline Cube $(001)[100]$ & & $: 4$ \\
\hline $\mathrm{CH} \quad(001)[120]$ & & 2.5 \\
\hline Brass (011)[211] & & 6.2 \\
\hline$S \quad(123)[634]$ & & $\beta$-fibre: 19.1 \\
\hline (112)[111] & & : 3.6 \\
\hline$(021)[100]$ & & : 3.8 \\
\hline$(001)[122]$ & & $: 2.4$ \\
\hline
\end{tabular}

intermediate and final batch annelings. The average $n$ value, measured by tensile test of several samples (averaged also in respect to the measured direction: the rolling and perpendicular to it), was 0.258 . The measurement of the strain rate sensitivity $m$, of $\mathrm{Al}-\mathrm{Mg}$ alloys is rather difficult task because of the serrated flow associated with $\mathrm{Mg}$ solute drag. By now the literature date for such alloys show either small negative or zero values. Since very accurate measurement facilities were not available the value used for the calculations was $m=0$. The alloy showed a recrystallization texture typical for aluminium alloys (Engler and Lücke, 1992) with Cuve, CH, CG, P and retained deformation $\beta$-fibre (Copper, Brass and " $S$ ") components. The volume fractions are listed in Table 1 .

\section{RESULT AND DISCUSSION}

\section{The influence of initial parameters on simulated FLD's}

In order to investigate the influence of the strain rate sensitivity on the FLD, a series of simulations with all parameters fixed except $m$, has been performed. The parameters were chosen as follow: $m=0,0.01,0.02,0.04, n=0.14$, texture "as measured" and "groove thickness" parameter $t_{b} / t_{a}=0.996$, (see below). The result is shown in Figure 1. The way $m$ affects the FLD can be summarised as follows:

- an increasing $m$-value shifts the FLD towards higher values of the major engineering strain $e_{1}$.

- an increasing $m$-value increase the plane strain region more than the stretching region (the slope of FLD in decreasing slightly in the stretching region with increasing $m$-value).

The way the FLD is affected by the strain hardening was studies by varying the $n$-value and keeping $m$ fixed at zero. The "groove thickness" parameter was 0.996 . The values used for $n$ were $0.32,0.25$ and 0.16 . The simulated FLD's are shown in Figure 2. Generally speaking the increase of $n$ shifts the whole FLD curve towards higher values of the major strain.

The initial groove thickness is represented by the parameter $t_{b} / t_{a}$, were $t_{b}$ is the thickness of the sheet in the groove and $t_{a}$ is the thickness out of it. This parameter was fixed during previous calculations on the value of 0.996 . Since an influence on calculated FLD's can be expected, a simulation with the same parameters but 


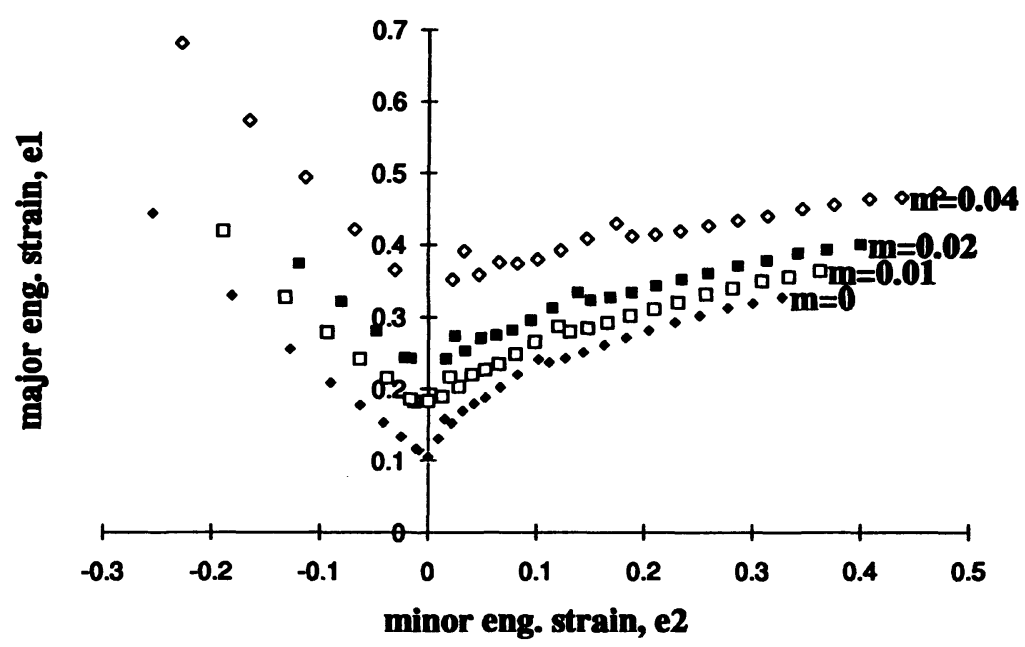

Figure 1 Influence of strain rate sensitivity exponent $m$ on calculated FLD (the other parameters were fixed at $n=0.14$, "groove thickness" $=0.996$, texture "as measured").

$t_{b} / t_{a}=0.99$ was performed and the comparison can be seen in Figure 3. There is a big difference between the two curves, mainly in the stretching region. Diminishing the value of the initial groove thickness (which means a bigger value of $t_{b} / t_{a}$ ) shifts the FLD towards higher values, increases the slope in the stretching region, but does not change the plane strain region much. In fact, the major objection to the MarciniakKuczynsky analysis is that the computational results are extremely sensitive to the size of the initial groove (Pishbin and Gillis, 1992). The value used for the groove thickness

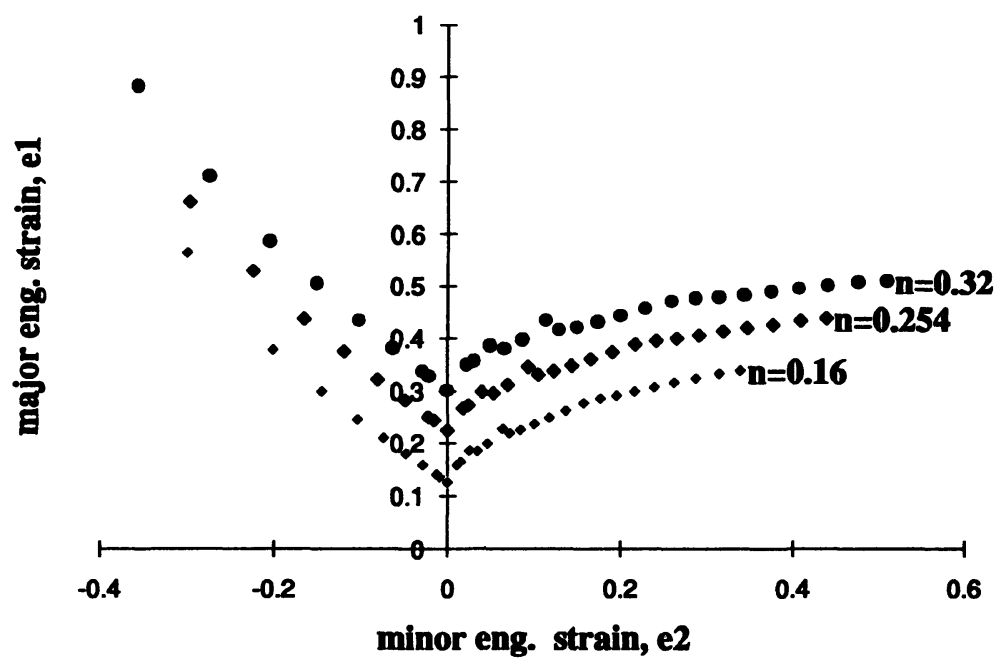

Figure 2 Influence of strain hardening parameter $n$ on calculated FLD (the other parameters were fixed at $m=0$, "groove thickness" $=0.996$, texture 'as measured'). 


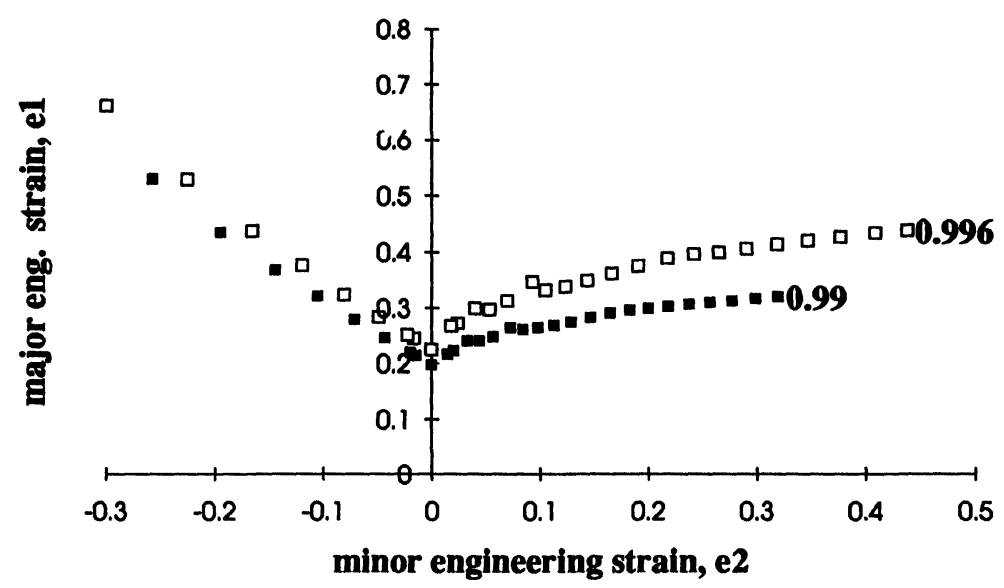

Figure 3 Influence of "groove thickness" parameter $t_{b} / t_{a}$ on calculated FLD (the other parameters were $m=0, n=0.14$, texture "as measured").

of 0.996 is recommended by model reasons, see for example Barlat (1987), and is not related to : $\mathrm{zal}$ physical properties. Without a precise estimation of the parameter a real fit of the simulated curves and the measured FLD's cannot be expected. It would certainly be important whether this parameter would be significantly influence by material properties and therefore would be different for different samples. Such influence might for example be expected from the grain size but no reliable literature date concerning this problems is known to the present authors. For comparative studies a fixed initial groove thickness (for instance the best fitting one) is recommended.

The influence of input texture was studied by comparing tow FLD's one simulated using the experimental texture and one generated from uniform texture, without prefer-

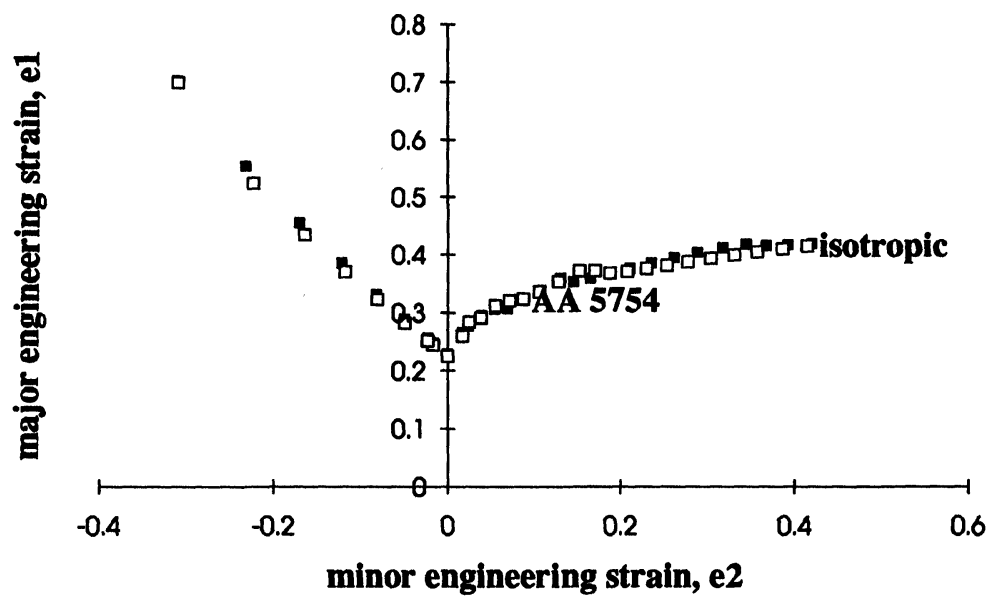

Figure 4 The calculated FLD, using experimental texture superimposed on the one generated with a uniform ("isotropic") texture. 
red orientations. Again all other parameters were kept fixed. The result is shown in Figure 4. The difference between the two curves is not significant. The result is well understandable if we take into account that the yield loci generated for these materials were not very different. In the region of interest, which ranges from the biaxial stretching point $\left(\varepsilon_{11}=\varepsilon_{22}\right)$ to the plane strain point $\left(\varepsilon_{22}=0\right)$, the yield loci based on measured textures have the same shape (but not necessarily the same size) of the yield locus of an isotropic material with the same slip system (see Figure 5). Note that the shape of these yield loci are quite different from those of b.c.c. metals, such as steel (because of the different slip systems), which in part explains the differences between the FLD's of steel and aluminium alloys. Another reason for the small texture contribution in the case of the aluminium alloy, studied in the present work is that the measured textures were generally rather weak.

\section{FLD simulations with ideal texture components}

In order to clarify the texture influence FLD's were simulated using artificial textures consisting of ideal texture components as input date, keeping all other simulation parameters fixed. The significant texture components experimentally observed in these alloys were used, namely:

- Cube (001)[100],

- CH (001)[120],

- H (001)[110] (rotated cube),

- (001)-fibre: combination of Cube, $\mathrm{H}$ and $\mathrm{CH}$ textures in equal parts,

- $\beta$-fibre: combination of "S", "Brass" and "Copper" components in a relative ratio as measured by texture.

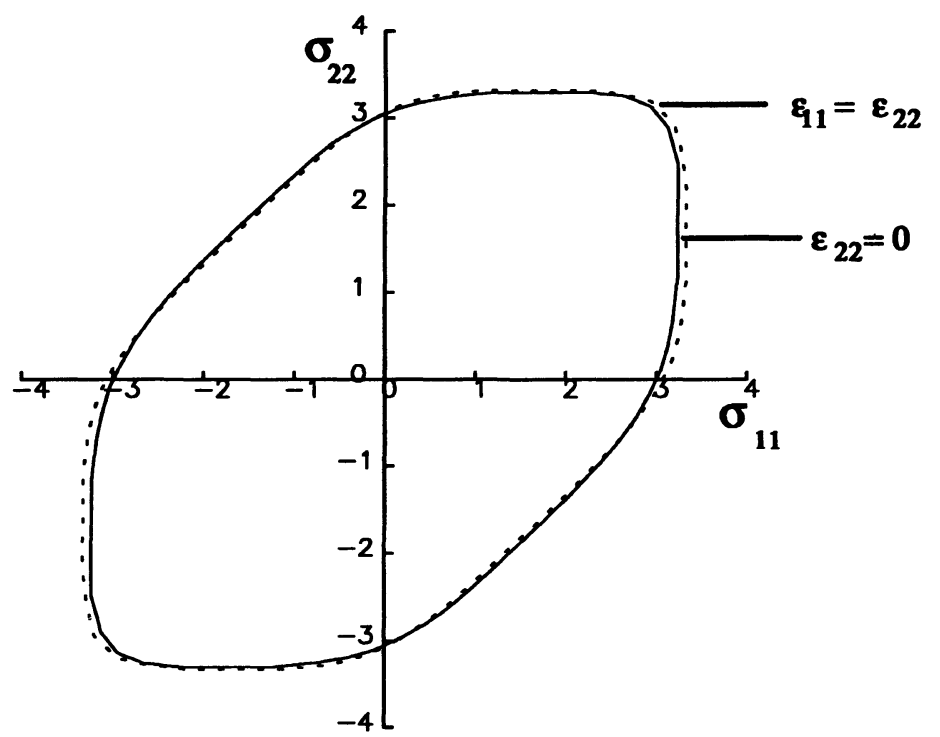

Figure 5 Yield loci derived from the texture of the alloy investigated and the one of a material with a "isotropic" texture (dotted line). The biaxial stretching point $\left(\varepsilon_{11}=\varepsilon_{22}\right)$ and the plane strain point $\left(\varepsilon_{11}=0\right)$ are indicated. 
The simulated FLD's are shown in Figure 6. The FLD for a uniform (isotropic) texture is also shown for reference. It is seen that:

- there is only a significant difference in the stretching region, $e_{2}>0$;

- referring to the isotropic texture the ideal texture components can be devided in two groups:

$=$ those with a positive influence (higher major strain at the same minor strain): Cube, $\mathrm{CH}$ and

$=$ textures with a negative influence: $H$ and $\beta$-fibre.

According to the model used for the calculations, on the right hand side of the curve the groove is supposed to appear perpendicular to the major strain direction (Van Houtte and Toth, 1993). The rolling direction (RD) in the above simulations is aligned to the major strain direction. FLD's have also been simulated for the case that the major strain and the rolling direction are not aligned. Technically, this is achieved by rotating the texture in the rolling plane (RP). Rotating Cube by $45^{\circ}$ leads to $\mathrm{H}$ ("rotated cube") and vice-versa. Together they constitute two cases: first: major strain axis parallel to the RD and to the [100] direction, second: same texture but the major strain axis at $45^{\circ}$ to the RD. The later case is " $\mathrm{H}$ " in Figure 6. According to Figure 6, the two cases give rather different FLD's. It means that although at first sight the Cube texture component seems to be very beneficial, the related formability is very anisotropic in the rolling plane - from highly positive (Cube) to rather negative influence (H-rotated at $45^{\circ}$ cube). So the cube texture does not appear very attractive because for major strain axis at $45^{\circ}(\mathrm{H}$ in Figure 6) there is a negative influence on formability. In the purely biaxial case $\left(\varepsilon_{11}=\varepsilon_{22}\right)$, the deformation itself is isotropic in the plane of the sheet. So the groove will choose the direction in which it propagates most easily. So, during sheet formation, there will be a low probability of crack formation perpendicular to the rolling direction, but a high one at $45^{\circ}$ to it.

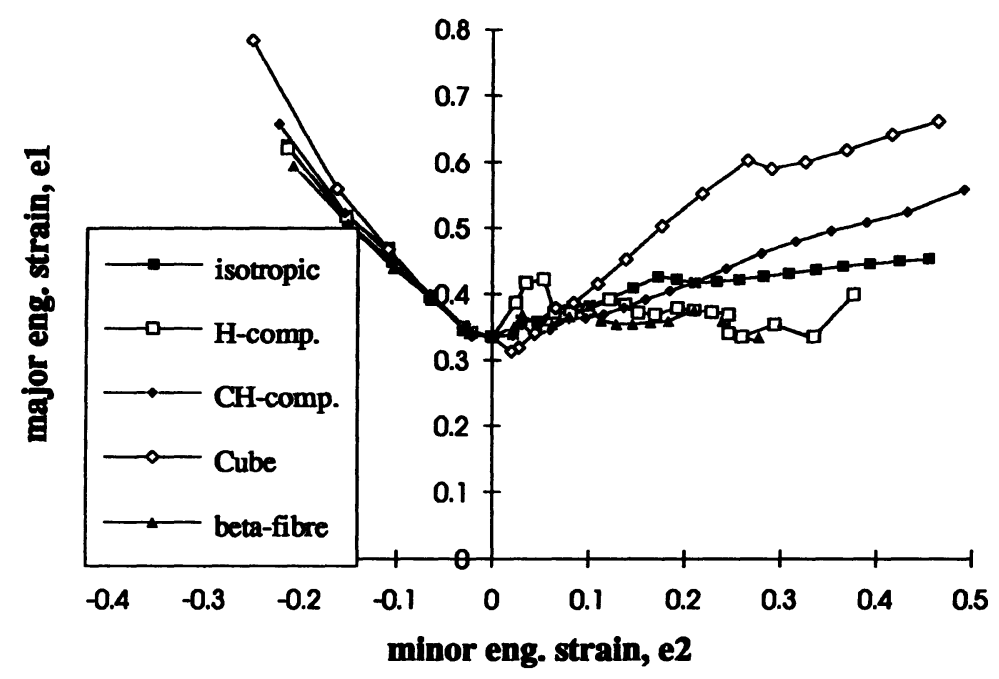

Figure 6 FLD's calculated by means of ideal input texture components superimposed on the one generated with the "isotropic' texture. 
Such a dependence of the simulated FLD on the angle between the major strain and the RD has been mentioned by Lin, Daniel and Jonas (1991), in the case of steel, and is qualified as "directionality" of texture influence on FLD. In fact such a directionality of the simulated FLD does not represent an observable value because only the minimum value of all possible limit strains is displayed by the real material. But in order to have a better fit with the experimental data this effect should be taken into account.

On Figure 7 the calculated FLD's of the $\mathrm{CH}$ and the rotated $45^{\circ} \mathrm{CH}$-fibre superimposed on the isotropic curve are shown. The $\mathrm{CH}$ texture gives a rather isotropic influence on the sheet formability. In the stretching region (especially for $e_{2}>0.2$ ) it is clearly positive. This texture component has shown the best formability (lowest anisotropy and highest FLD level) with the minimum effect at $0^{\circ}$ and the maximum effect at $45^{\circ}$. These effects (good formability for $\mathrm{CH}$ and high sensitivity to the major strain orientation for the Cube) have been found also by Lin et al. (1991). This result can be easily explain by comparing the 3 textures. On Figure 8a., b. and c. the $\varphi_{2}=0$ sections of the ODFs, generated with Cube, $\mathrm{H}$ and $\mathrm{CH}$ components are shown. A rotation of the $\mathrm{RD}$ with respect to the major strain axis is equivalent to a change of the angle $\varphi_{1}$. So, a $45^{\circ}$ of rotation of the beneficial Cube texture converts it into the harmful $\mathrm{H}$ texture. On the contrary, the $\mathrm{CH}$ texture when rotated at $45^{\circ}$ does not change much and, as a result, there is not much difference between the simulated FLD's. A $23^{\circ}$ rotation of this texture in the rolling plane gives a split on Cube and $\mathrm{H}$ textures that compensate each other regarding good or bad formability's (the FLD is between the ones with the Cube and the $\mathrm{H}$ components). This is the reason why this texture has a low anisotropy and is always beneficial for a sheet formability.

Sometimes a combination of Cube, $\mathrm{CH}$ and $\mathrm{H}$ textures is observed, namely the (001)fibre. A (001)-fibre generated by equal parts of the mentioned textures gives a $\varphi_{2}$ section as shown on Figure 8d. It is clear that such a texture will have a low in-plane anisotropy

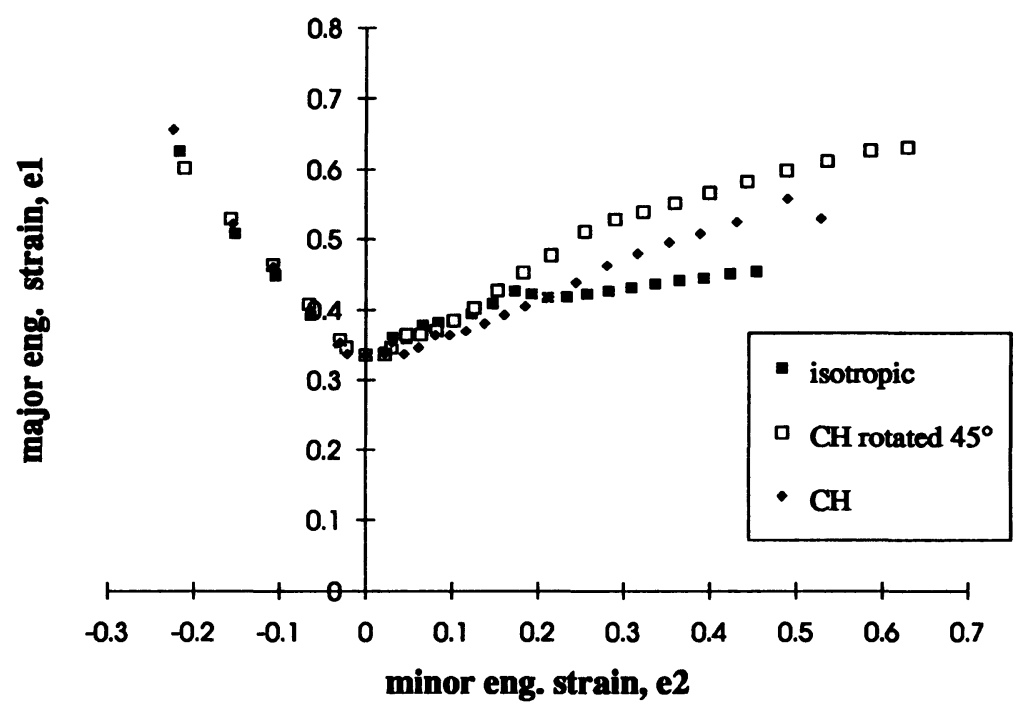

Figure 7 FLD's of the $\mathrm{CH}$ and $\mathrm{CH}$ texture components rotated at $45^{\circ}$ in comparison to the "isotropic" FLD. 


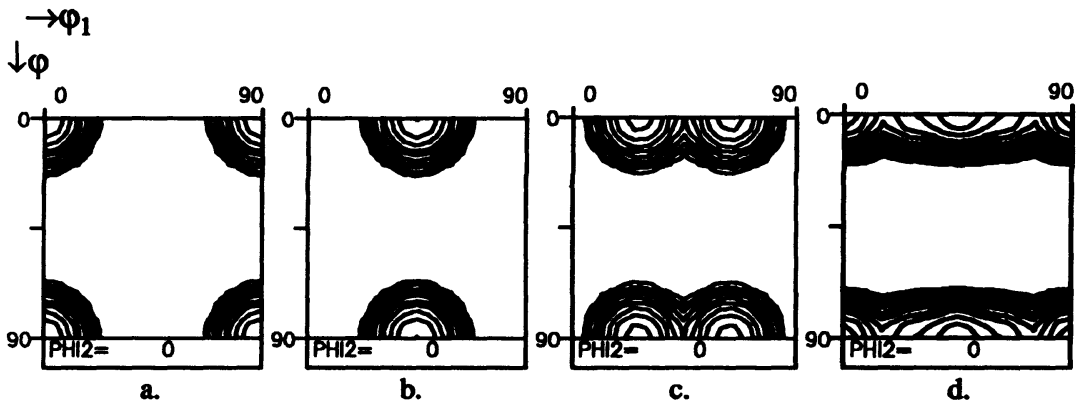

Figure $8 \varphi_{2}=0$ sections of the ODFs, generated with: a/Cube,

$\mathrm{b} / \mathrm{H}$,

c/CH components, d/(001)-fibre texture,

explaining why different texture components give in plane anisotropy in sheet forming.

of the simulated FLD's because the change of $\varphi_{1}$ does almost not change the texture. The Cube and the $\mathrm{H}$ texture will compensate each other regarding good or bad formability. On Figure 9, the calculated FLD's of the (001)-fibre (with Cube:CH:H $=1: 1: 1$ ) are shown. As expected, the (001)-fibre gives a FLD with a low anisotropy, very close to the FLD of the $\mathrm{CH}$ texture. It is clear that texture influences the sheet formability in a positive way.

The influence of the deformation texture ( $\beta$-fibre) on sheet formability has been simulated as well. A $\beta$-fibre consisting of " $S$ ", Copper and Brass components in the same proportions as in the measured texture was generated. The calculated FLD's with this input texture and the $30^{\circ}$ and $45^{\circ}$ rotated $R D$ superimposed on the isotropic FLD are shown on Figure 10 . The case of a $30^{\circ}$ rotated $\mathrm{RD}$ is considered here as well

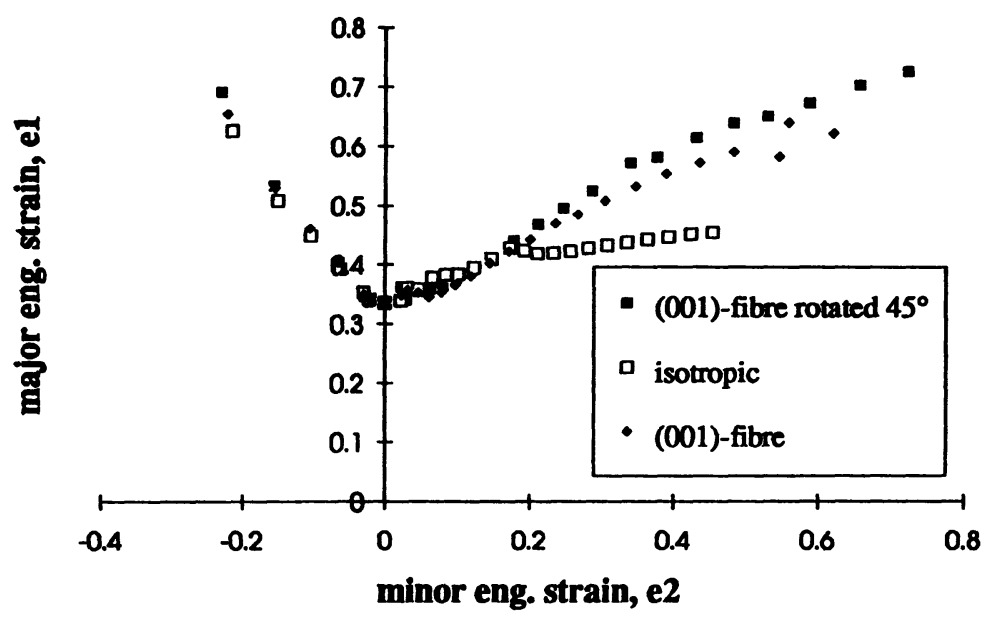

Figure 9 FLD's of (001)-fibre and rotated $45^{\circ}$ (001)-fibre superimposed on the "isotropic" FLD. Low anisotropy of the FLD and improved formability are obtained with this texture. 


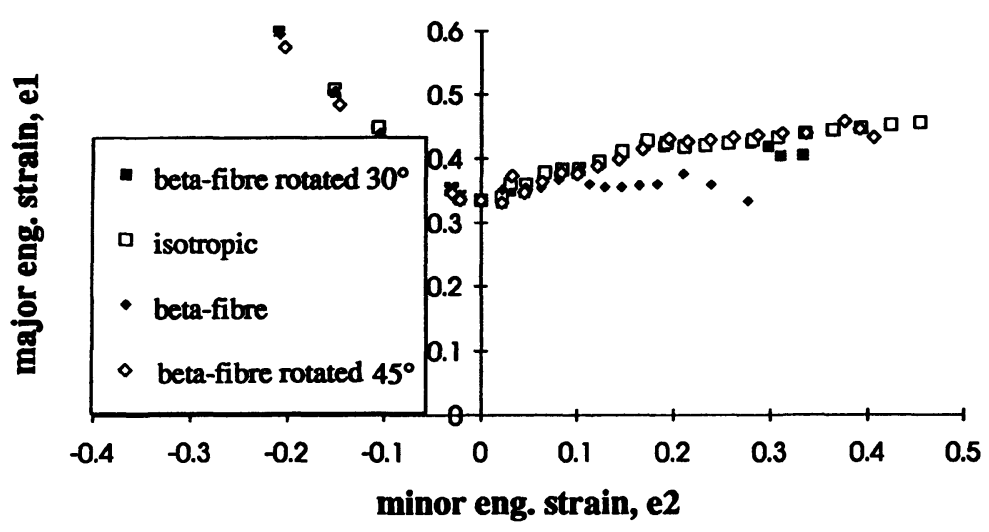

Figure 10 FLD's of the unrotated and rotated at $30^{\circ}$ and $45^{\circ} \beta$-fibre component in comparison to the "isotropic" FLD. It shows negative influence on formability.

because, according to Lin et al. (1991), most textures are responsible for either $0-45^{\circ}$ or $0-30^{\circ}$ "directionality and it cannot be stated in advance to which group the multicomponent $\beta$-fibre texture belongs. According to Figure 10 , the FLD with $45^{\circ}$ rotated RD shown a better formability, so for the $\beta$-fibre a $0-45^{\circ}$ anisotropy is valid as well. It can be seen that the $\beta$-fibre either has a negative influence on the sheet formability or almost no influence. Since the worst case must be retained, the $\beta$-fibre influence on formability is stated as negative.

\section{Comparison of simulated and experimentally measured FLD's}

The best criterion for the success of simulation is the comparison with experimentally measured curves. So, it would be of interest to compare simulated and measured FLD's. The simulated FLD were calculated by the use of the following parameters: $m=0$, $n=0.258$, "groove thickness" $=0.996$. The FLD, experimentally measured as described in the previous paragraph, is shown in Figure 11. The most obvious difference is that the minimum (the point with the smallest major strain) on the experimental curve is not at $e_{2}=0$. We have no explanation for that at present. In order to compare the two FLD's it is essential to shift the experimental one so that the minimum goes to $e_{2}=0$. From the curves aligned in this way (Figure 12) it can be seen that the measured FLD is considerably lower than the calculated one. Such a systematic difference between simulated and measured FLD's can be expected from the method of measuring the FLD's. Since measurement of strains are taken from circles next to the crack but not at the crack it is clear that the measured FLD's are underestimated. Approaching the crack develops a strain gradient, especially on the major strain direction. As a consequence, the measured FLD will depend on the grid diameter used. A curve published by Jalinier (1981) - Figure 13, is showing the difference between measured and real limit strain as a function of the diameter of the grid circles used. This dependence has been obtained by extrapolating to zero FLD date collected using different grid diameter (Grumbach and Sanz, 1972). This was done by the use of a theory that is not so appropriate for aluminium alloys, so this dependence can be used here only qualitatively. Nevertheless, it should be always taken into account that the measured FLD's are underestimated. 


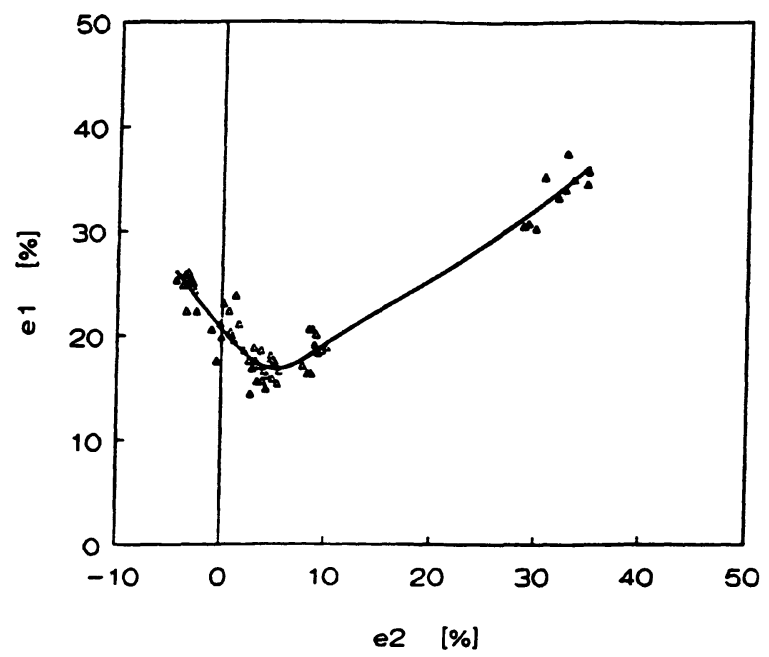

Figure 11 The FLD, experimentally measured for the samples investigated.

It should be noted that also the simulated FLD's incorporate some sources of discrepancy. Some of them are as follows:

- not all the initial parameters in the simulations are derived from direct measurements, for instance the $m$-value. The zero value used here might not be the right one. The value 0.996 used for the initial "groove thickness" parameter can also drastically change the curve. It is particularly unpleasant that it cannot be measured by physical

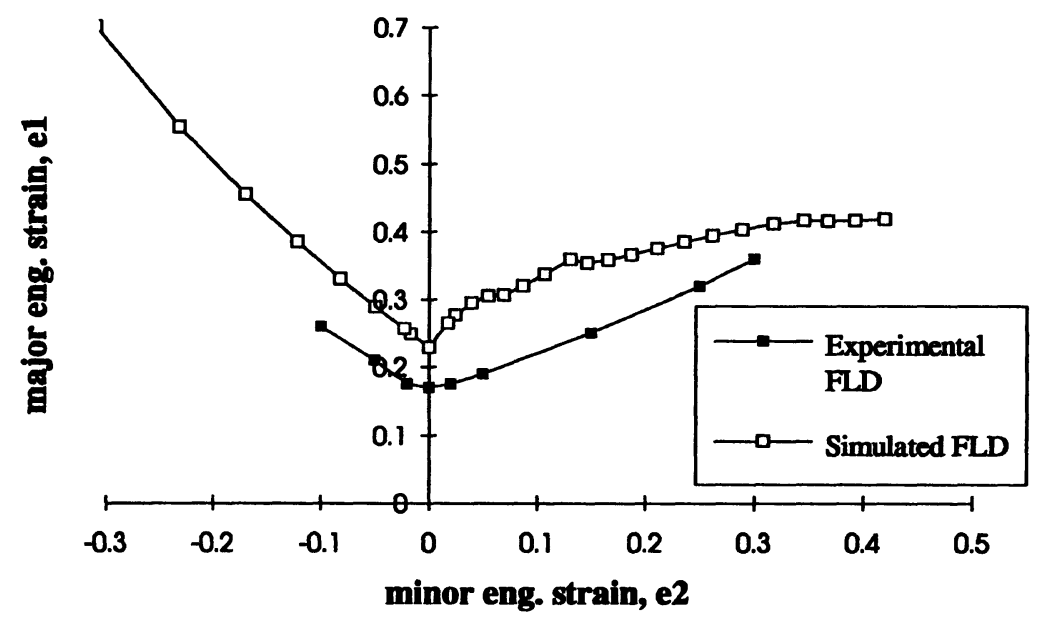

Figure 12 Comparison between the experimental (with a shifted zero position) and the simulated formability curves. The differences are due to

- first: underestimated experimental FLD and

- second: limited knowledge of initial parameters and model restrictions that affect the simulated FLD. 


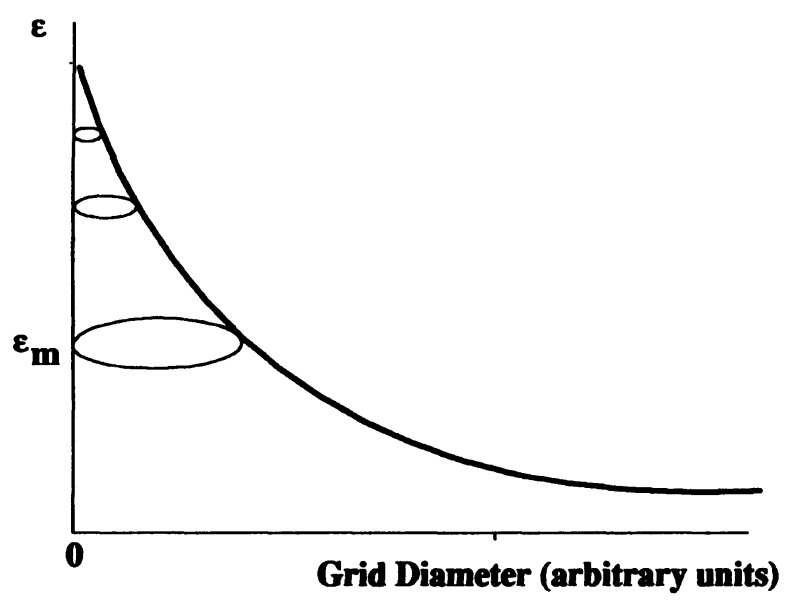

Figure 13 Difference between the measured and the real limit strain as a function of the grid circles diameter used, according to Jalineir (1981).

experiments. A way to solve this problem is to measure the $m$-value as well as possible and to vary the "groove thickness" parameter to the best fit.

- the change of the $m$-value, the $n$-value and the texture during the deformation is not taken into account by this model. It might have an additional influence. For the particular alloy investigated, the texture change will be most probably not important, but for the other input parameters this cannot be expected. An additional study is required to determine the influence of the evolution of $n$ and $m$ with strain.

\section{CONCLUSIONS}

The conclusions of the present work can be summarised as follows:

- increasing $m$-value shifts the FLD towards higher values of the major strain, especially in the plane strain region. The slope of FLD in the stretching region is slightly decreased;

- increasing $n$-value shifts the whole FLD curve towards higher values of the major strain;

- smaller values of the initial groove thickness (higher "groove thickness" parameter) shift the FLD towards higher values, increase the slope in the stretching region, but do not change the plane strain region much.

- there is no significant influence of the input texture on simulated FLD's for the alloy investigated, but in general the anisotropy should be considered;

- if strong textures are produced, a better formability of the alloy can be expected when a $\mathrm{CH}$ of a (001)-fibre texture is present. The Cube and the $\beta$-fibre texture have negative influence on formability.

- at this stage, the comparison between simulated and experimentally measured FLD's gives several differences: systematic - because of the underestimation of the limit strain in the experimental FLD, non-systematic - due to limited knowledge of the input parameters and model restrictions. 


\section{Acknowledgements}

The authors acknowledge Prof. S. Saimoto of Queen's University, Kingston, Canada for the valuable discussion about the nature of the strain rate sensitivity $m$ and some measurements of it and Dr. L. Tóth of the University of Metz, France for this many interesting discussions concerning FLD software.

\section{References}

1. Barlat, F. (1987). Material Science and Engineering, 91, 55.

2. Bunge, H. J. (1982). Texture Analysis in Materials Science, Butterworth Publishers, London.

3. Engler, O. and Lücke, K. (1992). S ripta Metallurgica et Materialia, 27, 1527.

4. Ferron, G. and Molinari, A. (1989). Forming Limit Diagrams: Concepts, Methods and Applications, Eds. Wagoner, R. H., Chan, R. S. and Keeler, S. P. The Minerals, Metals and Materials Society.

5. Graf, A. and Hosford, W. F. (1989). Forming Limit Diagrams: Concepts, Methods and Applications, Eds, Wagoner, R. H., Chan, R. S. and Keeler, S. P. The Minerals, Metals and Materials Society, p. 153.

6. Grumbach, M. and Sanz, G. (1972). Revue metallurgique, 69, 273.

7. Jalinier J. M. (1981). Mise en forme et endommagement. Thése d'Etat, Université de Metz, p. 27.

8. Lin, D. W., Daniel, D. and Jonas, J. J. (1991). Metallurgical Transactions A, 22A, 2069.

9. Marciniak, Z. and Kuczynski, K. (1967). Int. J. Mech. Sci, 9, 609.

10. Pishbin, H. and Gillis, P. P. (1992). Metallurgical Transactions A, 23A 2817.

11. Taylor, B. (1985). Metals Handbook, Ninth Edition, Vol. 8, Sheet Formability Testing, Eds. ASM Handbook Commitee, American Society for Metals Parks. Ohaio, p. 547.

12. Van Houtte, P., Mols, K., Van Bael, A. and Aernoudt, E. (1989)..Textures and Microstructure, 11, 23.

13. Van Noutte, P. and Tóth, L. S. (1993). Advances in Engineering Plasticity and its Application, Ed. Lee, W. B., Elsevier Science Publishers B.V. 